Louisiana State University

LSU Digital Commons

$10-8-2009$

\title{
Thermodynamics of the quantum critical point at finite doping in the two-dimensional Hubbard model studied via the dynamical cluster approximation
}

\author{
K. Mikelsons \\ Louisiana State University \\ E. Khatami \\ Louisiana State University \\ D. Galanakis \\ Louisiana State University \\ A. MacRidin \\ Fermi National Accelerator Laboratory \\ J. Moreno \\ Louisiana State University
}

See next page for additional authors

Follow this and additional works at: https://digitalcommons.Isu.edu/physics_astronomy_pubs

\section{Recommended Citation}

Mikelsons, K., Khatami, E., Galanakis, D., MacRidin, A., Moreno, J., \& Jarrell, M. (2009). Thermodynamics of the quantum critical point at finite doping in the two-dimensional Hubbard model studied via the dynamical cluster approximation. Physical Review B - Condensed Matter and Materials Physics, 80 (14) https://doi.org/10.1103/PhysRevB.80.140505

This Article is brought to you for free and open access by the Department of Physics \& Astronomy at LSU Digital Commons. It has been accepted for inclusion in Faculty Publications by an authorized administrator of LSU Digital Commons. For more information, please contact ir@lsu.edu. 


\section{Authors}

K. Mikelsons, E. Khatami, D. Galanakis, A. MacRidin, J. Moreno, and M. Jarrell 


\section{San Jose State University}

From the SelectedWorks of Ehsan Khatami

October, 2009

\section{Thermodynamics of the quantum critical point at finite doping in the two-dimensional Hubbard model studied via the dynamical cluster approximation}

K. Mikelsons, Louisiana State University

Ehsan Khatami, Louisiana State University

D. Galanakis, Louisiana State University

A. Macridin, Fermilab

J. Moreno, Louisiana State University, et al. 


\title{
Thermodynamics of the quantum critical point at finite doping in the two-dimensional Hubbard model studied via the dynamical cluster approximation
}

\author{
K. Mikelsons,${ }^{1,2}$ E. Khatami, ${ }^{1,2}$ D. Galanakis, ${ }^{1}$ A. Macridin, ${ }^{3}$ J. Moreno, ${ }^{1}$ and M. Jarrell ${ }^{1}$ \\ ${ }^{1}$ Department of Physics and Astronomy, Louisiana State University, Baton Rouge, Louisiana 70803, USA \\ ${ }^{2}$ Department of Physics, University of Cincinnati, Cincinnati, Ohio 45221, USA \\ ${ }^{3}$ Fermilab, P.O. Box 500, Batavia, Illinois 60510, USA
}

(Received 27 July 2009; revised manuscript received 26 August 2009; published 8 October 2009)

\begin{abstract}
We study the thermodynamics of the two-dimensional Hubbard model within the dynamical cluster approximation. We use continuous time quantum Monte Carlo as a cluster solver to avoid the systematic error which complicates the calculation of the entropy and potential energy (double occupancy). We find that at a critical filling, there is a pronounced peak in the entropy divided by temperature, $S / T$, and in the normalized double occupancy as a function of doping. At this filling, we find that specific heat divided by temperature, $C / T$, increases strongly with decreasing temperature and kinetic and potential energies vary like $T^{2} \ln T$. These are all characteristics of quantum critical behavior.
\end{abstract}

DOI: 10.1103/PhysRevB.80.140505

PACS number(s): 71.10.Fd, 71.10.Hf, 74.72.-h

\section{INTRODUCTION}

The properties of the hole-doped cuprate phase diagram, including a pseudogap (PG) at low doping and unusual metallic behavior at higher doping, have led many investigators to propose that there is a quantum critical point $(\mathrm{QCP})$ in the cuprate phase diagram at optimal doping. ${ }^{1}$ Some of the most compelling evidence for the QCP is from various thermodynamic experiments. ${ }^{2}$

In the previous work ${ }^{3}$ employing the dynamical cluster quantum Monte Carlo (QMC) method ${ }^{4,5}$ to calculate the quasiparticle fraction for the two-dimensional (2D) Hubbard model, we found evidence for a QCP separating a region of Fermi liquid (FL) character at high doping from a region of non-Fermi liquid PG character at low doping. At the critical doping, we found marginal Fermi liquid (MFL) character ${ }^{6}$ which is also seen above the FL and PG temperatures.

In this Rapid Communication, we provide further evidence for a QCP in the thermodynamic properties, including the energies, the specific heat and entropy. At the critical filling, both the kinetic and potential energy data show a $T^{2} \ln T$ low-temperature dependence, leading to a $T \ln T$ specific heat. The entropy divided by temperature, $S / T$, is strongly peaked at the critical point. In contrast to the results of the previous study, ${ }^{3}$ these quantities are independent of the location and character of the Fermi surface and thus avoid ambiguity in their interpretation.

\section{FORMALISM}

We start with the 2D Hubbard Hamiltonian

$$
H=H_{k}+H_{p}=\sum_{\mathbf{k} \sigma} \epsilon_{\mathbf{k}}^{0} c_{\mathbf{k} \sigma}^{\dagger} c_{\mathbf{k} \sigma}+U \sum_{i} n_{i \uparrow} n_{i \downarrow},
$$

where $c_{\mathbf{k} \sigma}^{\dagger}\left(c_{\mathbf{k} \sigma}\right)$ is the creation (annihilation) operator for electrons of wave vector $\mathbf{k}$ and spin $\sigma, n_{i \sigma}=c_{i \sigma}^{\dagger} c_{i \sigma}$ is the number operator, $\epsilon_{\mathbf{k}}^{0}=-2 t\left(\cos \left(k_{x}\right)+\cos \left(k_{y}\right)-2\right)$ with $t$ being the hopping amplitude between nearest-neighbor sites, and $U$ is the on-site Coulomb repulsion. The primary focus of this Rapid Communication is the behavior of the energies. For lack of a better term, we will refer to the quadratic part of $H$ as the kinetic energy and the interaction part as the potential energy. The corresponding energies per site are $^{7}$

$$
\begin{gathered}
E_{k}=\frac{\left\langle H_{k}\right\rangle}{N}=\frac{T}{N} \sum_{\omega_{n}, \mathbf{k}, \sigma} \epsilon_{\mathbf{k}}^{0} G_{\sigma}\left(\mathbf{k}, i \omega_{n}\right), \\
E_{p}=\frac{\left\langle H_{p}\right\rangle}{N}=\frac{T}{2 N} \sum_{\omega_{n}, \mathbf{k}, \sigma} \Sigma_{\sigma}\left(\mathbf{k}, i \omega_{n}\right) G_{\sigma}\left(\mathbf{k}, i \omega_{n}\right),
\end{gathered}
$$

where $G_{\sigma}\left(\mathbf{k}, i \omega_{n}\right)$ and $\Sigma_{\sigma}\left(\mathbf{k}, i \omega_{n}\right)$ are the single-particle Green's function and self-energy, respectively, with $\omega_{n}$ $=(2 n+1) \pi T$ and $N$ represents the number of sites.

Aside from the numerical calculations, we obtained analytically the leading low- $T$ behavior for $E_{k}$ and $E_{p}$ for each of the regions around the QCP starting from the corresponding forms for the self-energy. In the FL region, the imaginary part of the self-energy has the form

$$
\Sigma_{F L}^{\prime \prime}(\omega)=-\alpha_{F L} \max \left(\omega^{2}, T^{2}\right), \quad|\omega|<\omega_{X},
$$

where $\omega_{X}$ is a cutoff frequency and $\alpha_{F L}>0$. In the MFL region, this quantity becomes ${ }^{6}$

$$
\Sigma_{M F L}^{\prime \prime}(\omega)=-\alpha_{M F L} \max (|\omega|, T), \quad|\omega|<\omega_{c}
$$

where $\alpha_{M F L}>0$, and $\omega_{c}$ is a frequency cutoff. Finally in the PG region, we consider the following Ansatz ${ }^{8}$ for the imaginary part of the self-energy:

$$
\Sigma_{P G}^{\prime \prime}(\omega)=-\pi \Delta^{2}(\mathbf{k}) \delta\left(\omega-\epsilon_{\mathbf{k}}^{0}\right), \quad|\omega|<\omega_{c}^{*},
$$

where $\omega_{c}^{*}$ is a cutoff frequency and $\Delta(\mathbf{k})=\Delta_{0}\left(\cos k_{x}\right.$ $-\cos k_{y}$ ) with the constant $\Delta_{0}$ being the PG magnitude.

Inserting forms (4)-(6) in Eqs. (2) and (3) and performing a low- $T$ expansion in the resulting integrals, we obtained that both $E_{p}$ and $E_{k}$ exhibit a leading $T^{2}$ low-temperature behavior in the PG and FL regions. Both energies display a $T^{2} \ln T$ behavior consistent with a $T^{2} \ln T$ total energy ${ }^{9}$ in the MFL region. 


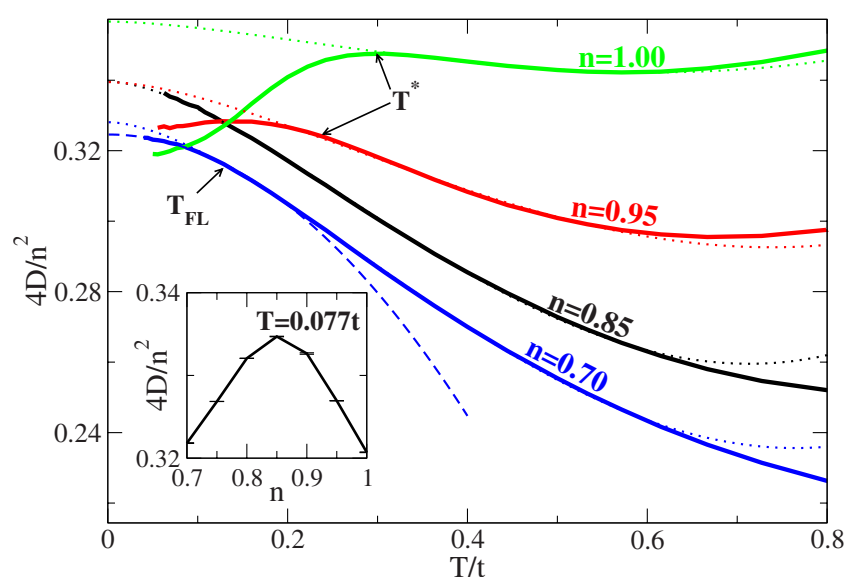

FIG. 1. (Color online) Temperature dependence of normalized double occupancy $4 D / n^{2}$. For a range of temperatures and values of filling, $D(T)$ fits well the MFL form (dotted lines): $D(T)=D_{0}$ $+\alpha T^{2} \ln \left(T /{ }_{c}\right)\left(D_{0}, \alpha\right.$, and ${ }_{c}$ are fitting parameters $)$. At low temperatures, $D(T)$ shows FL behavior (dashed line) for $n=0.70$ $\left(D(T)=D_{0}+\alpha T^{2}, \alpha \quad 0\right)$ and PG behavior for $n>0.85$. Indicated by arrows is the crossover temperature $T^{*}=0.13 t$ for $n=0.70$ along with the crossover temperatures $T_{F L}=0.24 t$ and $T_{F L}=0.3 t$ for $n$ $=0.95$ and $n=1.00$, respectively. For $n=0.85$, marginal Fermi liquid behavior persists down to lowest temperatures accessible with QMC. Inset: Normalized double occupancy versus filling for $T$ $=0.077 t$, showing a peak at the critical filling $n=0.85$.

\section{METHODOLOGY}

We solve the Hubbard model within the dynamical cluster approximation (DCA). ${ }^{4}$ The DCA is a cluster mean-field theory which maps the original lattice model onto a periodic cluster of size $N_{c}=L_{c}^{2}$ embedded in a self-consistent host. Spatial correlations up to a range $L_{c}$ are treated explicitly, while those at longer length scales are described at the meanfield level. However the correlations in time, essential for quantum criticality, are treated explicitly for all cluster sizes. To solve the cluster problem we use weak-coupling expansion continuous time QMC (CTQMC) method ${ }^{10,11}$ with highly optimized blocked and delayed updates. ${ }^{12}$ In the previous work, ${ }^{3}$ a Hirsch-Fye QMC (HFQMC) algorithm was used. However, the Trotter error intrinsic to HFQMC, which is particularly large for the potential energy (double occupancy), prevented us from calculating $S$ since systematic errors from multiple temperatures tend to accumulate. ${ }^{13}$ The CTQMC algorithm eliminates the systematic error, and generally has a lower overall computational cost than HFQMC, making it a better choice for these calculations. Unless otherwise displayed, the statistical error bars in these calculations are smaller than the plotting symbols or line widths used.

Here, we study the normal state on a $N_{c}=4 \times 4$ site cluster for $U=6 t$. We confirmed that increasing the cluster size to $N_{c}=24$ sites does not significantly alter our results. We obtain the energies from Eqs. (2) and (3), and, following Ref. 14 , the entropy from

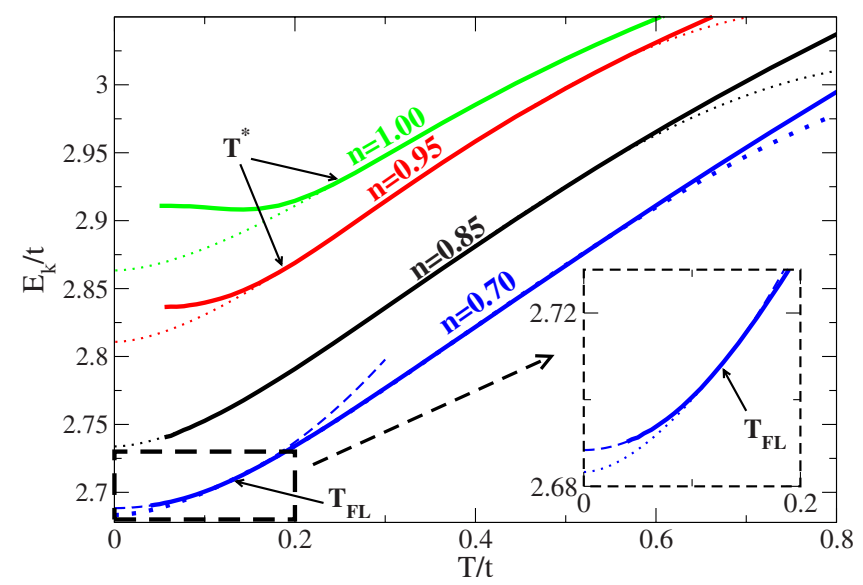

FIG. 2. (Color online) Temperature dependence of kinetic energy. MFL form fits data for a range of intermediate temperatures (dotted lines). For $n=0.70$, crossover to FL behavior is indicated as $T_{F L}$, while for $n=0.95$ and $n=1.00$, crossover to PG region at $T^{*}$ is evident by strong departure of kinetic energy from MFL fit, which for $n=1.00$ even results in noticeable increase of kinetic energy with lowering temperature.

$$
S(\beta, n)=S(0, n)+\beta E(\beta, n)-\int_{0}^{\beta} E\left(\beta^{\prime}, n\right) d \beta^{\prime},
$$

where $S(0, n)=-n \ln \frac{n}{2}-(2-n) \ln \left(1-\frac{n}{2}\right), n$ is the filling and $\beta=1 / T$. Finally, since the DCA preserves thermodynamic consistency, ${ }^{15}$ our results obey the Maxwell relation

$$
\left(\frac{\partial S}{\partial n}\right)_{T, U}=-\left(\frac{\partial \mu}{\partial T}\right)_{U, n},
$$

where $\mu$ is the chemical potential.

\section{RESULTS}

Figure 1 shows the normalized double occupancy, $4 D / n^{2}$, as a function of temperature at fillings $n=1.00,0.95,0.85$, and 0.70 together with fits to the MFL form for $n=0.95$, 0.85 , and 0.70 , and the FL form for $n=0.70$. For $n=0.95$ and in a low-temperature window, the $E_{p}$ first increases with decreasing temperature and then reaches a maximum at a temperature which coincides with the PG temperature $\left(T^{*}\right)$ found in a previous study. ${ }^{3}$ Further decreasing the temperature into the PG phase, $E_{p}$ deviates from the MFL character and begins to decrease. This indicates that close to half-filling, where the interactions are more relevant, the system tries to gain energy by lowering the $E_{p}$. A more pronounced decrease in the $E_{p}$ can be seen below the PG temperature in the halffilled case. At the critical filling, $n=0.85, E_{p}$ fits the MFL analytic form very well down to the lowest temperature reached. At this temperature, we find that the normalized double occupancy is maximal at the critical filling (see the inset of Fig. 1). For $n=0.70$, the $E_{p}$ increases with decreasing temperature as in the MFL region. However, we find that below the FL characteristic temperature $\left(T_{F L}\right)$ (see Ref. 3), it deviates from the MFL form and fits better to a quadratic function in $T$. Note that at intermediate temperatures $\left(T^{*}\right.$ or 


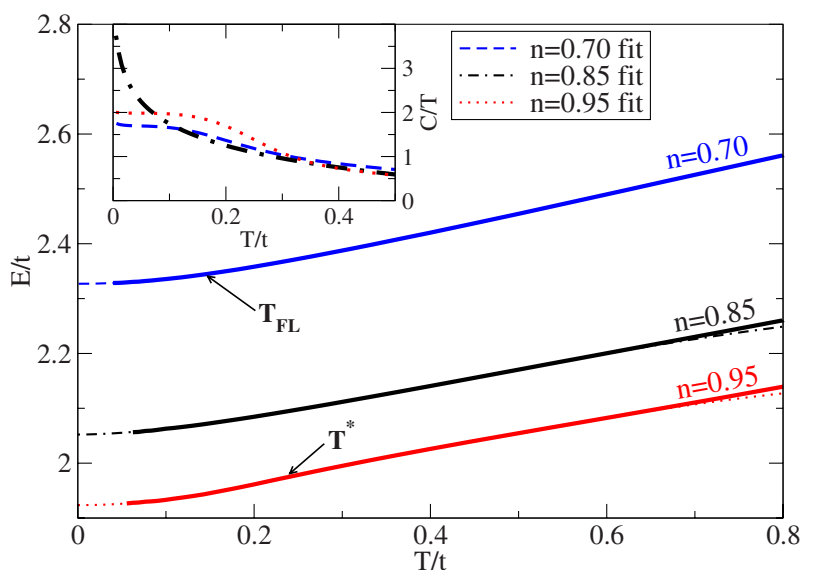

FIG. 3. (Color online) Total energy per site $E$ versus temperature for different fillings. The data are fit to a crossover form of the energy [Eq. (9) (dashed lines)]. The values of $T_{X}$ determined from the fit are indicated as $T_{F L}$ for $n=0.70$ and $T^{*}$ for $n=0.95$. In the inset, the specific heat calculated from the fit is plotted versus temperature.

$\left.T_{F L} \quad T \quad 0.6 t\right)$, the MFL form fits the $E_{p}$ very well in all three doping regions.

In Fig. 2, we show $E_{k}$ versus temperature at the same fillings. $T_{F L}$ and $T^{*}$ in Fig. 2 represent the onset of FL and PG regions obtained from $E_{k}$ fits, and they have the same value as those shown in Fig. 1 for the $E_{p}$. At low temperatures, while the $E_{k}$ deviates from its MFL fit for $n=1.00$, 0.95 , and 0.70 , one sees no sign of a characteristic temperature scale at the critical filling.

We note that the low- $T$ behavior of both $E_{p}$ and $E_{k}$ follows the analytical forms in the FL and MFL regions but slightly deviates from the predicted $T^{2}$ form in the PG region at low $T$. Also the energy scales below which the data deviate from the MFL $T^{2} \log T$ behavior vanishes at a critical filling, $n_{c}=0.85$, where it persists down to the lowest temperature accessible, consistent with the existence of a QCP between FL and PG regions.

The kinetic and potential energy data, together with the analytic forms of Eqs. (2) and (3) suggest that the total energy away from the half-filling may be fit to the form

$$
E(T)=E(0)+A f(T) T^{2}+B(1-f(T)) T^{2} \ln \frac{T}{\Omega},
$$

where $f(T)=1 /\left\{\exp \left[\left(T-T_{X}\right) / \theta\right]+1\right\}$ describes the crossover from the MFL to the quadratic behavior, characteristic of a FL or presumably PG region. $A, B, \theta, T_{X}$, and $\Omega$ are the fitting parameters of the QMC energy data, as shown in Fig. 3. At low $T$, the fit is indistinguishable from the data for all fillings. The values of $T_{X}$ determined from the fit are indicated as $T_{F L} \approx 0.15 t$ for $n=0.70$ and $T^{*} \approx 0.24 t$ for $n=0.95$ in agreement with the $E_{p}$ and $E_{k}$ fits. The calculation of the specific heat is a notoriously difficult problem and usually involves a fit of $E(T)$ to a regularized (smooth) functional form. ${ }^{16,17}$ Here, we already have an excellent fit, so we obtain $C / T$ from a derivative of the fit divided by temperature. The result is shown in the inset. As expected for $n=0.70$, at low temperatures, $C / T$ is flat in $T$ consistent with FL forma-

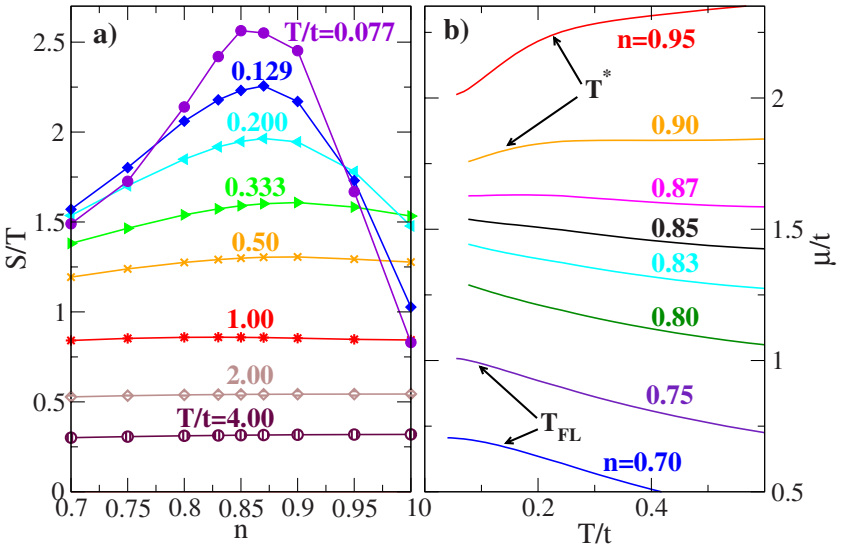

FIG. 4. (Color online) Left panel: $S / T$ filling dependence showing emergence of a peak at $n=0.85$ at low temperatures. Right panel: chemical potential temperature dependence for a range of fillings with PG and FL energy scales shown as $T^{*}$ and $T_{F L}$ for $n$ $=0.95$ and $n=0.70$, respectively. Note that the position of the maximum of entropy in the left panel corresponds to / $T=0$ in the right panel. As temperature is lowered, the maximum of entropy shifts toward lower $n$, causing local particle-hole symmetry for $n$ $=0.85$ at low $T$ (see text).

tion. This behavior is also seen for the data in the PG region, $n=0.95$, but the data at the critical filling $n=0.85$, shows a weak divergence at low $T$ consistent with quantum critical behavior.

The entropy per site exhibits intriguing behavior near the critical filling as the system is cooled, confirming much of the physics seen in $C / T$ without the need for a fit or a numerical derivative. It is more strongly quenched in the FL and PG regions than in the MFL region with decreasing $T$, resulting in a maximum in $S / T$ versus filling at $n=0.85$ at the lowest accessible temperature [see Fig. 4(a)]. The fact that $S / T$ continues to rise at the critical doping as $T \rightarrow 0$ is consistent with the increase in $C / T$. Similarly, the lowtemperature $S / T$ curves at different temperatures nearly overlap for $n \quad 0.85$ in agreement with a constant $C / T$ and indicative of a FL. One can see the strong decrease in $S / T$ with decreasing temperature when the system crosses over to the PG phase around $T^{*}$ at large fillings (e.g., at half-filling). In fact in the PG region, $n>0.85, S / T$ coincide for different temperatures only at one filling, roughly $n=0.95$. The flat low-T $C / T$ seen at this filling is accidental, and for higher filling one would expect $C / T$ to be strongly suppressed at low $T .^{18}$

According to Eq. (8), a local maximum in $S / T$ versus $n$ corresponds to a flat chemical potential as a function of temperature. Therefore, at low $T$, the critical filling can be identified from the temperature dependence of for different fillings. This is shown in Fig. 4(b) where, one can see that the near temperature independence of at $n=0.90$ for $0.25 t \quad T \quad 0.50 t$ evolves into a broad maximum centered around $T=0.15 t$ for $n=0.87$ which presumably moves to $n$ $=0.85$ at low enough temperatures. These observations are consistent with the evolution of the maximum in $S / T$ versus $n$ as the temperature is lowered from $0.50 t$ to $0.08 t$ [see Fig. 4(a)]. In analogy to the half-filled case, a stationary chemical 
potential can be the signature of local particle-hole symmetry. This is consistent with the observation of near particlehole symmetry in the cuprates in the proximity of optimal doping. ${ }^{19}$ It is also in agreement with previous results showing that at $n \approx 0.85$ and for the same model parameters, the low energy density of states displays particle-hole symmetry. ${ }^{3}$

\section{DISCUSSION}

The results presented here strongly favor an interpretation involving a QCP as opposed to a simple crossover from a FL to a non-Fermi liquid as the filling increases toward one. The $T^{2} \ln T$ behavior of the kinetic and potential energies, the peak in $S / T$ which sharpens as $T$ falls, and the logarithmic behavior of the specific heat $C / T \sim \ln T$ at $n=0.85$ together with the wide range of temperatures and fillings influenced by the critical point are clear signatures of quantum criticality.

The peak in the low-temperature normalized double occupancy observed at the critical filling is also interesting. Near half-filling, $4 D / n^{2}$ is suppressed by strong correlations. As the temperature is lowered $\left(\begin{array}{ll}T & T^{*}\end{array}\right)$, it is suppressed further to allow the system to gain the most magnetic exchange energy. As the filling decreases toward zero, the Fermi energy will become smaller than $U$, and again $4 D / n^{2}$ is suppressed. So for any finite $U$ we expect a peak in $4 D / n^{2}$ at some finite doping.

At the critical filling, in addition to the enhanced $C / T$, we find a peak in the charge susceptibility (not shown). These charge fluctuations may be responsible for the location of the peak $4 D / n^{2}$ near the critical doping. The peak in the charge susceptibility becomes sharper as the temperature is lowered, or when we include a next-near-neighbor hopping $t^{\prime}>0$. This behavior suggests the possibility that the QCP may be associated with a charge instability seen previously. ${ }^{20}$ This topic, as well as relation of the QPC and charge fluctuations to superconductivity, will be explored in an upcoming publication.

\section{CONCLUSION}

We study the thermodynamics of the 2D Hubbard model using the dynamical cluster approximation with CTQMC as a cluster solver. The latter eliminates the Trotter error which complicates the calculation of the entropy and potential energy. At the critical doping we find that $C / T$, obtained from a fit of $E(T)$, increases strongly with decreasing temperature and the kinetic and potential energies are consistent with $T^{2} \ln T$. Near the critical filling, we find a pronounced peak in $S / T$ which grows as the temperature falls, consistent with the growth of $C / T$. We also find a peak in the normalized double occupancy as a function of doping. These are all characteristics of quantum criticality.

\section{ACKNOWLEDGMENTS}

We would like to thank P. Phillips, S. Kivelson, D. J. Scalapino, A. M. Tremblay, and C. Varma for useful conversations. This research was supported by NSF Contract No. DMR-0706379. J.M. and M.J. are also supported by NSF PIRE Project No. OISE-0730290. This research used resources of the National Center for Computational Sciences at Oak Ridge National Laboratory, which is supported by the Office of Science of the U.S. Department of Energy under Contract No. DE-AC05-00OR22725.
${ }^{1}$ S. Sachdev and J. Ye, Phys. Rev. Lett. 69, 2411 (1992).

${ }^{2}$ C. Bernhard, J. L. Tallon, Th. Blasius, A. Golnik, and Ch. Niedermayer, Phys. Rev. Lett. 86, 1614 (2001), and references therein.

${ }^{3}$ N. S. Vidhyadhiraja, A. Macridin, C. Sen, M. Jarrell, and M. Ma, Phys. Rev. Lett. 102, 206407 (2009).

${ }^{4}$ M. H. Hettler, A. N. Tahvildar-Zadeh, M. Jarrell, T. Pruschke, and H. R. Krishnamurthy, Phys. Rev. B 58, R7475 (1998); M. H. Hettler, M. Mukherjee, M. Jarrell, and H. R. Krishnamurthy, ibid. 61, 12739 (2000)

${ }^{5}$ M. Jarrell, Th. Maier, C. Huscroft, and S. Moukouri, Phys. Rev. B 64, 195130 (2001).

${ }^{6}$ C. M. Varma, Phys. Rev. Lett. 83, 3538 (1999); C. M. Varma, Z. Nussinov, and W. van Saarloos, Phys. Rep. 361, 267 (2002).

${ }^{7}$ A. L. Fetter and J. D. Walecka, Quantum Theory of ManyParticle Systems (McGraw-Hill, New York, 1971).

${ }^{8}$ K.-Y. Yang, T. M. Rice, and F.-C. Zhang, Phys. Rev. B 73, 174501 (2006).

${ }^{9}$ M. Crisan and C. P. Moca, J. Supercond. 9, 49 (1996).

${ }^{10}$ S. M. A. Rombouts, K. Heyde, and N. Jachowicz, Phys. Rev. Lett. 82, 4155 (1999).

${ }^{11}$ A. N. Rubtsov, V. V. Savkin, and A. I. Lichtenstein, Phys. Rev. B
72, 035122 (2005).

${ }^{12}$ K. Mikelsons, A. Macridin, E. D’Azevedo, K. Tomko, and M. Jarrell (unpublished).

${ }^{13}$ A.-M. Daré, L. Raymond, G. Albinet, and A.-M. S. Tremblay, Phys. Rev. B 76, 064402 (2007).

${ }^{14}$ F. Werner, O. Parcollet, A. Georges, and S. R. Hassan, Phys. Rev. Lett. 95, 056401 (2005).

${ }^{15}$ Th. Maier, M. Jarrell, Th. Pruschke, and M. H. Hettler, Rev. Mod. Phys. 77, 1027 (2005).

${ }^{16}$ C. Huscroft, R. Gass, and M. Jarrell, Phys. Rev. B 61, 9300 (2000).

${ }^{17}$ A. K. McMahan, C. Huscroft, R. T. Scalettar, and E. L. Pollock, J. Comput.-Aided Mater. Des. 5, 131 (1998).

${ }^{18}$ This behavior in $S(T)$ at $n=1$ is likely an artifact of the DCA used here where the self-energy is not interpolated. An interpolated self-energy, as used in, e.g., Ref. 3, would preserve the d-wave character of the PG and the $T^{2}$ character of $E(T)$.

${ }^{19}$ S. Chakraborty, D. Galanakis, and P. Phillips, arXiv:0807.2854 (unpublished).

${ }^{20}$ A. Macridin, M. Jarrell, and Th. Maier, Phys. Rev. B 74, 085104 (2006). 